\title{
Astronomy in Colombia
}

\author{
William E. Cepeda-Peña ${ }^{1}$ \\ ${ }^{1}$ National Astronomical Observatory, National University of Colombia \\ email: wecepedap@unal.edu.co
}

\begin{abstract}
Astronomy in Colombia has been done since the beginning of the nineteenth century, when in 1803 one of the oldest (or maybe the oldest) astronomical observatories of America was built. This is a very beautiful, historical and ancient building. A small dome with a small telescope is also inside the university campus. The observatory marks since then the development of astronomy in Colombia as a professional science. At the present time a Master's programme and a Specialization programme are successfully carried out with a good number of smart young students. The observatory has a staff of eleven professors, all with a master's degree in sciences; two of them have a $\mathrm{PhD}$ and in a couple of years five staff members will have a $\mathrm{PhD}$ in physics. With some international collaboration, they will introduce in a few years a doctoral astronomical program. There are several research lines mainly in the fields of astrometry, Galactic and extragalactic astronomy, cosmology, astro-statistics and astrobiology. Three research groups have got recognition from the governmental institution that supports the research in sciences COLCIENCIAS. Several papers have been published in national and international journals. Besides the professional line in astronomy, the observatory sponsors several non-professional Colombian astronomical groups that work enthusiastically in the field of astronomy.
\end{abstract}

Keywords. Galactic astronomy, AGN, teaching of astronomy

\section{Historical background}

The National Astronomical Observatory of Colombia, NAO, founded in 1803, is the oldest permanent structure in the Americas that was designed for use of a fixed telescope for astronomical observations. The NAO was conceived by Jose Mutis and built with the intention that Caldas be director.

José Mutis is the central figure in early science in Colombia. Mutis was a physician named 'first botanist and astronomer' by King Carlos III for the Botanical Expedition of 1783, which was equipped with a Sisson quadrant, two Adams theodolites, and two Emery chronometers. They were supplied in Spain with a transit instrument, a telescope by Herschel and two achromatic telescopes, but these were lost in transit. At the College of Rosario, Mutis taught mathematics, physics and, against the mainstream Dominican teachings, he taught Copernican astronomy, possibly the first in America to do so. The site chosen was the garden of the Botanical Expedition. It is close to the equator, and in 1803 there was no other observatory at a higher elevation. The 56-foot octagonal tower, diameter 27 feet, has instrument rooms in successive stories. A second 'staircase' tower is 72 feet tall. There were telescopes in the upper rooms of both towers.

On May 24, 1802, the construction of the building began, and it was completed August 20, 1803. Caldas was appointed director in 1805, and in December 1805 began programmes of astronomical observation, meteorological projects, teaching local students, and he helped start a weekly scientific journal. During July of 1810 , political unrest in Colombia reached a critical point. Caldas was forced to abandon scientific work, become a military engineer, and engage in the production of maps and armaments. 
Francisco José de Caldas, was a lawyer with an interest in astronomy. Circa 1785, he began measuring meridians and calculating latitudes and azimuths. To mark solstices, Caldas measured the amplitude of the ecliptic. He made many meteorological and geographical measurements, including the determination of local longitude using published ephemerides and astronomical phenomena including lunar eclipses and occultations of stars and moons of Jupiter. For the lunar eclipse of 1797, he used an achromat of 30 inches focus to derive longitude.

The arrival of Alexander von Humboldt in Colombia gave a great impetus to science in the area. In 1801, Humboldt tutored Caldas in meteorology and astronomy, giving him star tables and catalogues, and teaching computation. Humboldt had a significant influence on Caldas the astronomer; instructing him on the use of tables of atmospheric refraction and the use of instruments. He provided Caldas with an octant and a quadrant, and star catalogues and ephemeris. Caldas observed the transit of Mercury of 9 November, 1802, timing the final two contact points.

Caldas was an advocate of independence from Spain and allowed activists to meet at the observatory. As a consequence of this, he was arrested by Spanish troops and executed in 1816. Caldas is a national hero to Colombians, probably a unique example of an observatory director elevated to heroic status. Julio Garavito became director in 1891, and despite being equipped with antiquated instruments, was active in both observation and theory. Studies included mathematical optics, lunar motion, Newtonian mechanics, and criticism of relativity. Further civil unrest caused problems at the National University, and engineering classes moved to the observatory. In 1949, Ruiz Wilches, directed the construction of a new observatory in 1952, in the campus of the National University of Colombia, NUC, with a 4-element apochromatic refractor, of $20 \mathrm{~cm}$ aperture and 3 metres focal length, previously used at the Observatory of Marseilles, France.

\section{Professional astronomers}

The NAO has a staff of eleven professors all with a master's degree in sciences; two of them are $\mathrm{PhD}$ and in a couple of years five staff members will have a $\mathrm{PhD}$ in physics. The Colombian astronomers received their training in the Science Faculty of NUC.

\section{Teaching of astronomy}

Although astronomy does not appear in the curriculum of the secondary schools, it is being taught in some universities. The teaching is mainly conducted by lectures and courses. The 'General Astronomy' course is an elective for students of the Physics Department of NUC. The NAO leads since then the development of astronomy in Colombia as a professional science. At the present time a Master's programme and a Specialization programme in astronomy are successfully carried out with a good number of smart young students. In the near future, the NAO plans to introduce a PhD programme in astronomy.

\section{Scientific interests}

Astronomical research in Colombia were chiefly concentrated in theoretical parts rather than the practical requirements of the country. The field of interest are:

- Fundamental astrometry. They are training at NAO.

- Celestial mechanics. The analytical theory of motion of Earth artificial satellites. They are studied at NAO. Some papers were published in this field. 
- Ephemerides. The main tasks were compiling the astronomical calendar and nautical almanac, predicting astronomical phenomena (eclipses, comets), and calculating astronomical elements as a service to different governmental institutions of Colombia.

- Active nuclei of galaxies, AGN.

- Black holes and cosmology.

- Galactic astronomy. A lot of papers were published in this field.

Colombian astronomers also wrote books on: Astronomical courses in general and special relativity; dissemination of astronomical knowledge for example: Astronomy for everyone, spherical astronomy and eclipses.

The Galactic Astronomy Group Stellar Clusters is the principal research group in the NAO. Research is being done in different topics by the Galactic Astronomy Group Stellar Clusters. In the field of open clusters, the research has been focused on membership working from proper motions and proper motions and positions; an astro-statistical approach has been used to obtain membership probabilities using mainly Bayesian theory and the EM or expectation maximization algorithm; the required software has been developed.

Another topic is galaxy groups and group lensing. Weak lensing is a powerful tool to test the large-scale structure of the universe. Due to the low signal, this effect is only a statistical one, but with a wide application that goes from the determination of the cosmological parameters of matter density, the cosmological constant or dark energy, normalization of the power spectrum, the Hubble constant, and to constrain shapes and sizes of the dark matter halos. We use weak lensing to study the dark matter distribution in galaxy groups, together with kinematics and dynamics of the galaxies in the group. We are developing new tools to extend the work made in galaxy clusters, using mainly the tangential shear as a signal of the matter distribution, and we compare our results with the dynamics and some tracers, such as inter-galactic matter.

Another line of research is dark energy and dark matter in galactic halos. The distribution of galaxies in the universe is one of the key astronomical observations (together with the cosmic background radiation and supernova Ia light-curves), which is used to identify the cosmological model that rules the dynamics of the universe. Such observations have shown that the universe is in an accelerated phase with zero curvature; these features indicate a dominance of an unknown relativistic fluid, the so called 'dark energy'. The cosmological constant has been claimed to be the best candidate to represent such a component, but still there are other candidates that also lead to the present universe. These are the so called 'Chaplygin gas', 'dynamical dark energy' or 'quintessence models' (inspired by inflationary models). Hence, the main goal of this line of research is to identify which of these candidates fits in the best way the observations of the galaxy distribution.

Finally, some astrometric work is also done in this research group, looking for the best description of the movement of the Sun in the solar neighborhood.

\section{References}

Bateman, A. 1953, El Observatorio Astronómico de Bogotá, Universidad Nacional de Colombia, Bogotá.

OAN, 1997 Plan de desarrollo Observatorio: Astronómico Nacional. Universidad Nacional de Colombia, Bogotá.

Velázquez, M. 1994, Caldas. Molinos Velásquez Editores, Bogotá. 


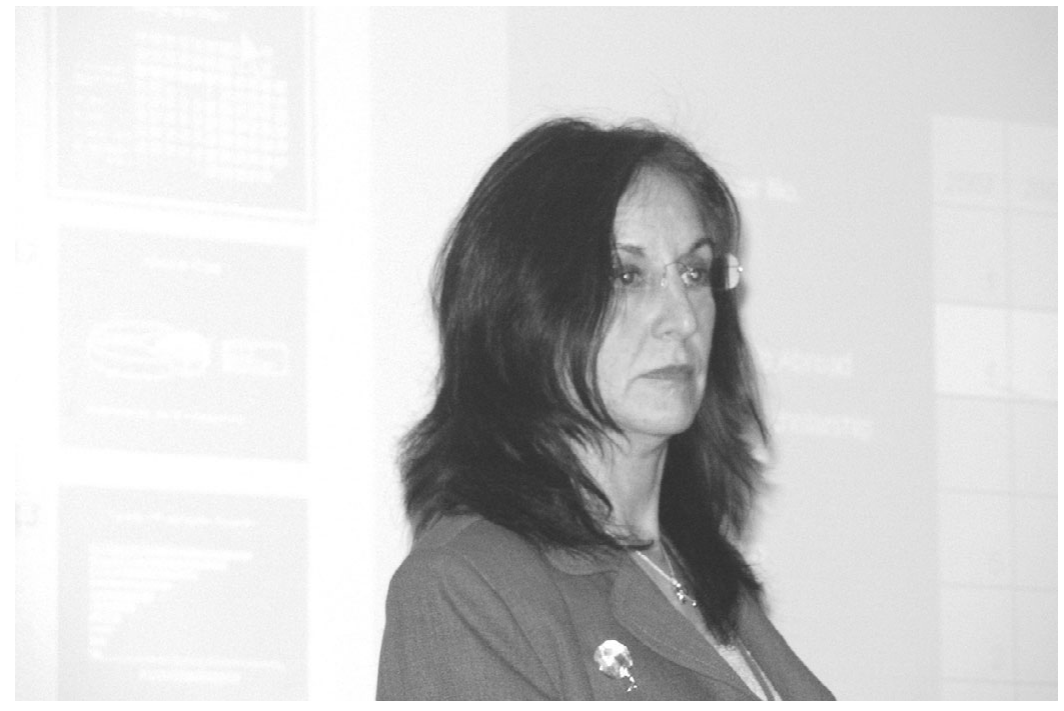

Julieta Fierro

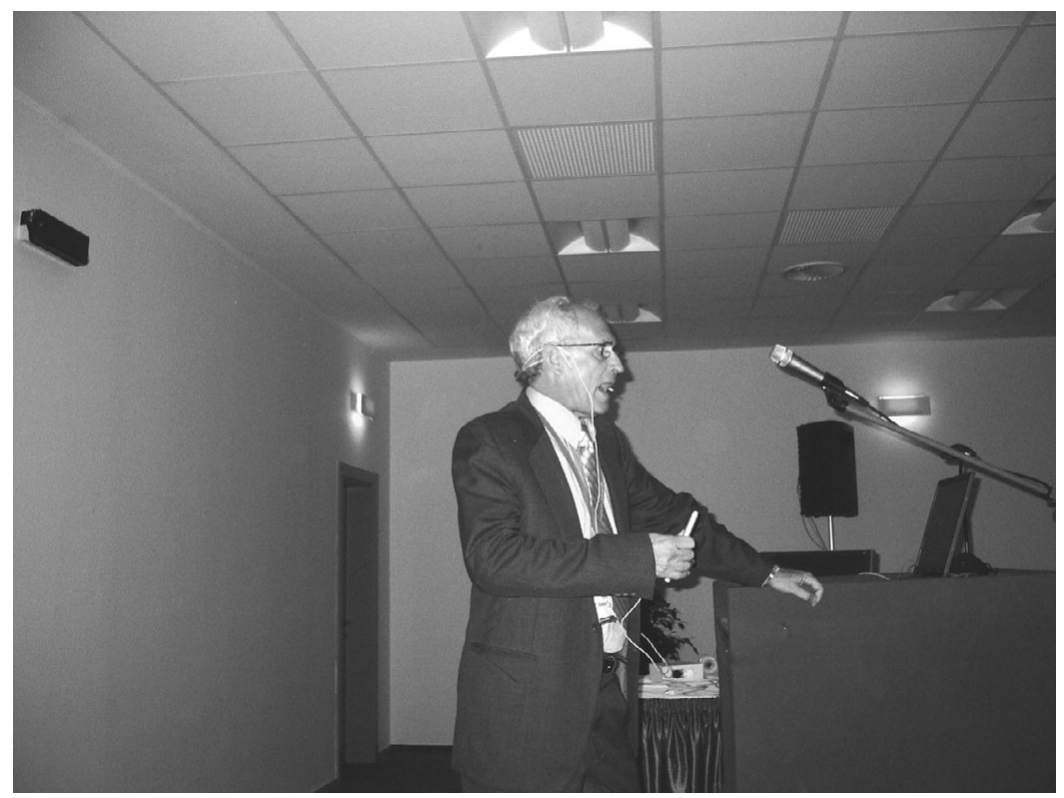

Oscar Álvarez 